\title{
CHARACTERIZATION OF THE MONOTONE POLAR OF SUBDIFFERENTIALS
}

\author{
MARC LASSONDE
}

\begin{abstract}
We show that a point is solution of the Minty variational inequality of subdifferential type for a given function if and only if the function is increasing along rays starting from that point. This provides a characterization of the monotone polar of subdifferentials of lower semicontinuous functions, which happens to be a common subset of their graphs depending only on the function.
\end{abstract}

Given a graph $T \subset X \times X^{*}$, we call monotone polar of $T$ the set

$$
T^{0}:=\left\{\left(x, x^{*}\right) \in X \times X^{*}:\left\langle y^{*}-x^{*}, y-x\right\rangle \geq 0, \quad \forall\left(y, y^{*}\right) \in T\right\} .
$$

The object of this note is to characterize the monotone polar of subdifferentials $\partial f$ of extended real-valued lower semicontinuous functions $f$ on a Banach space $X$.

We see from the above definition that a pair $(\bar{x}, 0)$ in $X \times X^{*}$ belongs to $(\partial f)^{0}$ if and only if $\bar{x}$ is solution of the Minty variational inequality of subdifferential type defined by:

$$
\sup \langle\partial f(y), \bar{x}-y\rangle \leq 0, \quad \forall y \in X .
$$

We are thus led to consider such systems of inequalities.

In Section 1, we study Minty variational inequalities of (lower Dini) subderivative type for functions $f$ on a convex subset $C \subset X$. We show that a point $\bar{x} \in C$ is solution of such a system if and only if $f$ is increasing on $C$ along rays starting from $\bar{x} \in C$. (This is a variant of [2, Theorem 2.1].) In Section 2, we consider the case of subdifferentials of functions $f$. These objects are simply set-valued mappings $\partial f: X \rightrightarrows X^{*}$ that lie between the subdifferential of convex analysis for $f$ and its Clarke subdifferential, and satisfy the so-called Separation Principle. Using the link established in [5] between subderivative and subdifferential, we show that Minty variational inequalities of subderivative type and of subdifferential type have the same set of solutions. Finally, in Section 3, we provide the desired characterization of the monotone polar of subdifferentials of functions $f$ : it consists in the set of all pairs $\left(x, x^{*}\right)$ in $X \times X^{*}$ such that the perturbed function $f-x^{*}$ is increasing on $X$ along rays starting from $x$. Therefore, all subdifferentials have the same polar, which is a common subset of their graphs.

\section{Minty Variational inequality of SUbDerivative type}

In the following, $X$ is a real Banach space with unit ball $B_{X}, X^{*}$ is its topological dual, and $\langle.,$.$\rangle is the duality pairing. For x, y \in X$, we let $[x, y]:=\{x+t(y-x): t \in[0,1]\}$, and $[x, y[$ is defined accordingly.

All the functions $f: X \rightarrow]-\infty,+\infty]$ are assumed to be lower semicontinuous and proper, which means that the set $\operatorname{dom} f:=\{x \in X: f(x)<\infty\}$ is nonempty. The (radial or lower

Date: July 6, 2013.

2010 Mathematics Subject Classification. Primary 49J52; Secondary 49K27, 26D10, $26 \mathrm{~B} 25$.

Key words and phrases. lower semicontinuity, subdifferential, lower Dini subderivative, Minty variational inequality, increase-along-rays property, monotone polar, maximal monotonicity. 
Dini) subderivative of a function $f$ at a point $\bar{x} \in \operatorname{dom} f$ is given by:

$$
\forall d \in X, \quad f^{\prime}(\bar{x} ; d):=\liminf _{t \searrow 0} \frac{f(\bar{x}+t d)-f(\bar{x})}{t} .
$$

Here is a simple version of the mean value inequality for extended real-valued lower semicontinuous functions in terms of the subderivative (for a proof, see, e.g., [4, 5]):

Mean Value Inequality. Let $X$ be a Banach space, $f: X \rightarrow]-\infty,+\infty]$ be lower semicontinuous, $\bar{x} \in X$ and $x \in \operatorname{dom} f$. Then, for every real number $\lambda \leq f(\bar{x})-f(x)$, there exists $x_{0} \in\left[x, \bar{x}\left[\right.\right.$ such that $\lambda \leq f^{\prime}\left(x_{0} ; \bar{x}-x\right)$.

The next proposition asserts that, given a function $f$ and a convex set $C \subset X$, a point $\bar{x} \in C$ is solution of the Minty variational inequality of subderivative type associated to $f$ on $C$ if and only if $f$ is increasing on $C$ along rays starting from $\bar{x}$. It is a variant of [2, Theorem 2.1].

Proposition 1.1. Let $X$ be a Banach space, $f: X \rightarrow]-\infty,+\infty]$ be lower semicontinuous, $C \subset X$ be convex and $\bar{x} \in C$. Then, the following are equivalent:

(a) $f^{\prime}(y ; \bar{x}-y) \leq 0$ for every $y \in C$,

(b) $f(y+t(\bar{x}-y)) \leq f(y)$ for every $y \in C$ and $t \in[0,1]$.

Proof. We show that $\neg(\mathrm{b})$ implies $\neg(\mathrm{a})$. Assume there exist $y \in C$ and $\bar{y} \in[y, \bar{x}]$ such that $f(\bar{y})>f(y)$. Applying the above Mean Value Inequality with $0<\lambda<f(\bar{y})-f(y)$, we find $y_{0} \in[y, \bar{y}[\subset C$ such that

$$
f^{\prime}\left(y_{0} ; \bar{y}-y\right) \geq \lambda>0 .
$$

Since $\bar{x}-y_{0}=\tau(\bar{y}-y)$ for some $\tau>0$, we have $f^{\prime}\left(y_{0} ; \bar{x}-y_{0}\right)=\tau f^{\prime}\left(y_{0} ; \bar{y}-y\right)>0$, proving that $\neg$ (a) holds.

Conversely, we show that (b) implies (a). Let $y \in C$ and assume $f(y+t(\bar{x}-y)) \leq f(y)$ for every $t \in[0,1]$. Then,

$$
\limsup _{t \searrow 0} \frac{f(y+t(\bar{x}-y))-f(y)}{t} \leq 0
$$

a fortiori, $f^{\prime}(y ; \bar{x}-y) \leq 0$.

\section{Minty Variational IneQuality of SubdiffERential tyPe}

The subdifferential of a lower semicontinuous function $f$ in the sense of convex analysis at a point $\bar{x} \in \operatorname{dom} f$ is the subset of $X^{*}$ defined by

$$
\partial_{c x} f(\bar{x}):=\left\{x^{*} \in X^{*}:\left\langle x^{*}, y-\bar{x}\right\rangle+f(\bar{x}) \leq f(y), \forall y \in X\right\} .
$$

The Clarke subdifferential [1] of $f$ at $\bar{x} \in \operatorname{dom} f$ is given by

$$
\partial_{C} f(\bar{x}):=\left\{x^{*} \in X^{*}:\left\langle x^{*}, d\right\rangle \leq f^{\uparrow}(\bar{x} ; d), \forall d \in X\right\},
$$

where

$$
f^{\uparrow}(\bar{x} ; d):=\sup _{\delta>0} \limsup _{\substack{t \searrow^{t} 0 \\ x \rightarrow f}} \inf _{d^{\prime} \in d+\delta B_{X}} \frac{f\left(x+t d^{\prime}\right)-f(x)}{t} .
$$

It is easily seen that $\partial_{c x} f(\bar{x}) \subset \partial_{C} f(\bar{x})$ with equality whenever $f$ is convex lower semicontinuous. 
In what follows, we call subdifferential any operator $\partial$ that associates a set-valued mapping $\partial f: X \rightrightarrows X^{*}$ to each function $f$ on $X$ so that $\partial f$ lies between $\partial_{c x} f$ and $\partial_{C} f$ :

$$
\partial_{c x} f \subset \partial f \subset \partial_{C} f
$$

and satisfies the elementary property $\partial\left(f-x^{*}\right)(x)=\partial f(x)-x^{*}$ for all $x \in X$ and $x^{*} \in X^{*}$. We also require that subdifferentials satisfy the following basic calculus rule on the Banach space $X$ :

Separation Principle. For any lower semicontinuous functions $f, \varphi$ on $X$ with $\varphi$ convex Lipschitz near $\bar{x} \in \operatorname{dom} f \cap \operatorname{dom} \varphi$, if $f+\varphi$ admits a local minimum at $\bar{x}$, then $0 \in \partial f(\bar{x})+\partial \varphi(\bar{x})$.

Examples. The Clarke subdifferential and the Ioffe subdifferential satisfy the Separation Principle in any Banach space. The limiting versions of the basic elementary subdifferentials (proximal, Fréchet, Hadamard) satisfy the Separation Principle in appropriate Banach spaces. All these subdifferentials also satisfy the inclusions (2.1) For more details, see, e.g., [3, 4] and the references therein.

Here is the link between subderivative and subdifferential. It involves the following $\varepsilon$ enlargement of the subdifferential:

$$
\breve{\partial}_{\varepsilon} f(\bar{x}):=\left\{x_{\varepsilon}^{*} \in X^{*}: x_{\varepsilon}^{*} \in \partial f\left(x_{\varepsilon}\right) \text { with }\left\|x_{\varepsilon}-\bar{x}\right\| \leq \varepsilon,\left|f\left(x_{\varepsilon}\right)-f(\bar{x})\right| \leq \varepsilon,\left\langle x_{\varepsilon}^{*}, x_{\varepsilon}-\bar{x}\right\rangle \leq \varepsilon\right\} .
$$

Subderivative/Subdifferential Inequality [5]. Let $X$ be a Banach space, $f: X \rightarrow]-\infty,+\infty$ ] be lower semicontinuous and $\bar{x} \in \operatorname{dom} f$. Then, for every $\varepsilon>0$, the sets $\breve{\partial}_{\varepsilon} f(\bar{x})$ are nonempty and

$$
\forall d \in X, \quad f^{\prime}(\bar{x} ; d) \leq \inf _{\varepsilon>0} \sup \left\langle\breve{\partial}_{\varepsilon} f(\bar{x}), d\right\rangle
$$

Injecting Formula (2.2) into Proposition 1.1 enables us to show that Minty variational inequalities of subderivative type and of subdifferential type on an open convex set have actually the same set of solutions. This complements our previous result [5, Theorem 3.3] on optimality conditions in terms of subdifferentials.

Theorem 2.1. Let $X$ be a Banach space, $f: X \rightarrow]-\infty,+\infty]$ be lower semicontinuous, $U \subset X$ be open convex and $\bar{x} \in U$. Then, the following are equivalent:

(a) $\sup \langle\partial f(y), \bar{x}-y\rangle \leq 0$ for every $y \in U$,

(b) $f(y+t(\bar{x}-y)) \leq f(y)$ for every $y \in U$ and $t \in[0,1]$.

Proof. We show that $\neg$ (b) implies $\neg($ a). Assume there exist $y \in U$ and $\bar{y} \in[y, \bar{x}]$ such that $f(\bar{y})>f(y)$. Then, by Proposition 1.1 there exists $y_{0} \in U$ such that $f^{\prime}\left(y_{0} ; \bar{x}-y_{0}\right)>0$. Let $\varepsilon>0$ such that $f^{\prime}\left(y_{0} ; \bar{x}-y_{0}\right)>\varepsilon$ and $y_{0}+\varepsilon B_{X} \subset U$, and apply Formula (2.2) at point $y_{0}$ and direction $d=\bar{x}-y_{0}$ to obtain a pair $\left(y_{\varepsilon}, y_{\varepsilon}^{*}\right) \in \partial f$ such that

$$
\left\|y_{\varepsilon}-y_{0}\right\|<\varepsilon, \quad\left\langle y_{\varepsilon}^{*}, y_{\varepsilon}-y_{0}\right\rangle<\varepsilon, \quad\left\langle y_{\varepsilon}^{*}, \bar{x}-y_{0}\right\rangle>\varepsilon .
$$

Hence, $y_{\varepsilon} \in U$ and $y_{\varepsilon}^{*} \in \partial f\left(y_{\varepsilon}\right)$ satisfy $\left\langle y_{\varepsilon}^{*}, \bar{x}-y_{\varepsilon}\right\rangle>0$, which proves $\neg$ (a).

Conversely, let us assume that $f\left(y^{\prime}+t\left(\bar{x}-y^{\prime}\right)\right) \leq f\left(y^{\prime}\right)$ for every $y^{\prime} \in U$ and $t \in[0,1]$. We have to show that $\sup \langle\partial f(y), \bar{x}-y\rangle \leq 0$ for every $y \in U$. Let $y \in U$. We claim that $f^{\uparrow}(y ; \bar{x}-y) \leq 0$. Indeed, let $\delta>0$ such that $\left.\left.y+\delta B_{X} \subset U, t \in\right] 0,1\right]$ and $y^{\prime} \in y+\delta B_{X}$. Then

$$
\inf _{d^{\prime} \in \bar{x}-y+\delta B_{X}} \frac{f\left(y^{\prime}+t d^{\prime}\right)-f\left(y^{\prime}\right)}{t} \leq \frac{f\left(y^{\prime}+t\left(\bar{x}-y^{\prime}\right)\right)-f\left(y^{\prime}\right)}{t} \leq 0 .
$$


It follows that, for any $\delta>0$,

$$
\limsup _{\substack{t \searrow 0 \\ y^{\prime} \rightarrow f_{f} y}} \inf _{d^{\prime} \in \bar{x}-y+\delta B_{X}} \frac{f\left(y^{\prime}+t d^{\prime}\right)-f\left(y^{\prime}\right)}{t} \leq 0,
$$

hence, $f^{\uparrow}(y ; \bar{x}-y) \leq 0$. This proves the claim. We derive that

$$
\sup \left\langle\partial_{C} f(y), \bar{x}-y\right\rangle \leq f^{\uparrow}(y ; \bar{x}-y) \leq 0
$$

so also $\sup \langle\partial f(y), \bar{x}-y\rangle \leq 0$ since $\partial f \subset \partial_{C} f$. This completes the proof.

\section{Monotone polar of SUbdifferentials}

Given a set-valued operator $T: X \rightrightarrows X^{*}$, or graph $T \subset X \times X^{*}$, we let

$$
T^{0}:=\left\{\left(x, x^{*}\right) \in X \times X^{*}:\left\langle y^{*}-x^{*}, y-x\right\rangle \geq 0, \forall\left(y, y^{*}\right) \in T\right\}
$$

be the set of all pairs $\left(x, x^{*}\right) \in X \times X^{*}$ monotonically related to $T$. As in [6], we call monotone polar of $T$ the operator $T^{0}: X \rightrightarrows X^{*}$, or graph $T^{0} \subset X \times X^{*}$, thus defined. An operator $T: X \rightrightarrows X^{*}$ is said to be monotone provided $T \subset T^{0}$, maximal monotone provided $T=T^{0}$ and monotone absorbing provided $T^{0} \subset T$.

In [5] we proved that all subdifferentials of lower semicontinuous functions, not only the convex ones, are monotone absorbing. Below we show a more precise statement: the monotone polar of any subdifferential $\partial f$ is precisely the set of all pairs $\left(x, x^{*}\right) \in X \times X^{*}$ such that the perturbed function $f-x^{*}$ is increasing along all rays starting from $x$. Thus, all subdifferentials of lower semicontinuous functions have the same monotone polar and are monotone absorbing.

Theorem 3.1. Let $X$ be a Banach space and let $f: X \rightarrow]-\infty,+\infty]$ be proper lower semicontinuous. Then, for every $x \in X$,

$$
(\partial f)^{0}(x)=\left\{x^{*} \in X^{*}:\left(f-x^{*}\right)(y+t(x-y)) \leq\left(f-x^{*}\right)(y), \forall y \in X, t \in[0,1]\right\} .
$$

Proof. Let $x^{*} \in(\partial f)^{0}(x)$. This means that $\left\langle y^{*}-x^{*}, y-x\right\rangle \geq 0$ for every $\left(y, y^{*}\right) \in \partial f$. Since $\partial\left(f-x^{*}\right)(y)=\partial f(y)-x^{*}$, this can be rewritten as

$$
\sup \left\langle\partial\left(f-x^{*}\right)(y), x-y\right\rangle \leq 0 \quad \text { for every } y \in X,
$$

which is equivalent by Theorem 2.1 to

$$
\left(f-x^{*}\right)(y+t(x-y)) \leq\left(f-x^{*}\right)(y) \quad \text { for every } y \in X \text { and } t \in[0,1] .
$$

The proof is complete.

\section{REFERENCES}

[1] F. H. Clarke. Optimization and nonsmooth analysis, volume 5 of Classics in Applied Mathematics. Society for Industrial and Applied Mathematics (SIAM), Philadelphia, PA, second edition, 1990.

[2] G. P. Crespi, I. Ginchev, and M. Rocca. Minty variational inequalities, increase-along-rays property and optimization. J. Optim. Theory Appl., 123(3):479-496, 2004.

[3] A. D. Ioffe. On the theory of subdifferentials. Adv. Nonlinear Anal., 1(1):47-120, 2012.

[4] F. Jules and M. Lassonde. Subdifferential estimate of the directional derivative, optimality criterion and separation principles. Optimization, http://dx.doi.org/10.1080/02331934.2011.645034.

[5] F. Jules and M. Lassonde. Subdifferential test for optimality. J. Global Optim., http://dx.doi.org/10.1007/s10898-013-0078-6.

[6] J.-E. Martínez-Legaz and B. F. Svaiter. Monotone operators representable by l.s.c. convex functions. SetValued Anal., 13(1):21-46, 2005.

Université des Antilles et de la Guyane, 97159 Pointe À Pitre, France

E-mail address: marc.lassonde@univ-ag.fr 